\section{Rheumatic disease and COVID-19: epidemiology and outcomes}

\author{
Kimme L. Hyrich $(1)$ and Pedro M. Machado $(0)$
}

\begin{abstract}
Since the outset of the COVID-19 pandemic, numerous risk factors for severe disease have been identified. Whether patients with rheumatic diseases, especially those receiving DMARDs, are at an increased risk of SARS-CoV-2 infection or severe COVID-19 disease remains unclear, although epidemiological studies are providing some insight.
\end{abstract}

The COVID-19 pandemic, caused by SARSCoV-2 infection, has seen over 71 million confirmed cases and over 1.6 million deaths worldwide recorded up to 15 December 2020, although the true number of cases worldwide is unknown ${ }^{1}$. For most people, COVID-19 will cause a mild-to-moderate flu-like illness characterized by fever, cough, and loss of taste and smell, among other symptoms; for some patients, however, the disease takes a severe and aggressive form, requiring hospitalization and ventilatory support, and potentially results in death. Whether or not patients with pre-existing immune-mediated inflammatory diseases (IMIDs), such as rheumatic diseases, are at an increased risk of SARS-CoV-2 infection or of severe COVID-19 outcomes remains unclear. Over the past 6 months, an unprecedented number of case series and reports of COVID-19 in patients with rheumatic diseases have been published; in this Year in Review commentary we highlight three of the larger studies published over this period of the pandemic ${ }^{2-4}$, which have advanced our knowledge of the risk of COVID-19 in this population (FIG. 1).

Using data from the new OpenSAFELY electronic platform, holding the primary care health records of $\sim 17$ million adults in England, Williamson et al. ${ }^{2}$ examined factors associated with 10,926 COVID-19-related deaths $(0.06 \%$ of the study population) over the first 3 months of the pandemic. They observed that people with a diagnosis of rheumatoid arthritis (RA), systemic lupus erythematosus (SLE) or psoriasis, analysed as a combined group (5.1\% of the study population), were (slightly) more likely to die in relation to COVID-19 compared to people without one of these diagnoses; this finding persisted after allowing for any variation in age, sex, ethnicity, social deprivation and the presence of other chronic health conditions (adjusted HR 1.19; 95\% CI 1.11-1.27). This observation is important but is limited by the lack of more specific knowledge of the diseases at the individual level, a common limitation of primary care data. Rheumatic diseases are highly heterogeneous, and thus information on current levels of disease activity, specific disease-related comorbidities and, most critically, the use of glucocorticoids and/or DMARDs, all of which are themselves risk factors for serious infection ${ }^{5}$, is needed to further understand what is driving this increased risk of COVID-19-related death. The investigators also did not have access to data on the prevalence of SARS-CoV-2 infection in the population, so it is not known whether patients with these conditions are at increased risk of infection or of dying if infected. Although tempting, causal interpretations of the OpenSAFELY findings should be avoided.

The potential role of DMARDs in the presentation, severity and even management of COVID-19 has received considerable attention. At the outset of the pandemic, hydroxychloroquine was touted as both a preventive and therapeutic treatment for COVID-19, but subsequent clinical trials have not found any benefit ${ }^{6}$. Many cytokines, such as TNF and IL-6, are involved in both the physiological response as well as the pathological response (for example, the 'cytokine storm') seen with COVID-19 (REF.). Cytokine inhibitors used in rheumatic diseases are therefore of interest, in terms of whether they are effective treatments for severe COVID-19 and whether their chronic use in patients with rheumatic diseases might alter the course of infection. However, the role of a cytokine storm in COVID-19-induced organ dysfunction has been questioned, as the extent of cytokinaemia in cases of severe and critical COVID-19 is less than that seen in other disorders associated with elevated cytokine production, such as chimeric antigen receptor (CAR) $\mathrm{T}$ cell-induced cytokine release syndrome and non-COVID-19 acute respiratory distress syndrome ${ }^{8}$.

To explore whether cytokine inhibitors could modulate the risk of infection, Simon et al. ${ }^{3}$ undertook a study looking at SARS-CoV-2 seroprevalence among 793 patients with IMIDs in Bavaria, Germany, 534 of whom were receiving cytokine inhibitors and 359 of whom were not, as well as 971 healthy individuals and 285 health-care professionals. Overall, the prevalence of seroconversion was very low; only 46 people $(2.2 \%$ of the total cohort) tested positive for anti-SARS-CoV-2 IgG antibodies. Compared with healthy individuals, the rate of positivity did not differ in patients with IMIDs not receiving cytokine inhibitors (relative risk (RR) 1.21; 95\% CI $0.50-2.90$ ) but was significantly lower among those who were receiving them (RR 0.32; 95\% CI 0.11-0.99). Patients with IMIDs were less likely to have traveled or been in contact with an infected person than were the healthy individuals, but these behaviours were not related to use of cytokine inhibitors, suggesting the possibility that, in some way, these therapies might reduce susceptibility to COVID-19. A correlation between SARS-CoV-2 IgG antibody titre and severity of COVID-19 has also been reported ${ }^{9}$, which could suggest that patients receiving cytokine inhibitors might have been exposed to SARS-CoV-2 infection but did not develop symptomatic illness. Unfortunately, the number of cases in the Simon et al. ${ }^{3}$ study was too low to draw any more specific conclusions on the role of anti-cytokine therapies in COVID-19 disease severity, and therefore caution must be taken to not overinterpret these data, as there were in fact no significant differences (overlapping confidence intervals) in seroconversion rates between patients with IMIDs receiving and not receiving cytokine inhibitors.

\footnotetext{
Key advances

- Patients with rheumatoid arthritis, systemic lupus erythematosus or psoriasis, when analysed as a combined group, might have a slightly increased risk of death from COVID-19 compared to those without these diseases, although the role of disease activity and treatment in this risk estimation was not taken into account ${ }^{2}$.

- Treatment with cytokine inhibitors could reduce the risk of SARS-SoV-2 infection (as measured by development of SARS-CoV-2 antibodies), although the mechanisms of this protective effect are not clear ${ }^{3}$.

- Chronic use of glucocorticoids at moderate or high doses ( $\geq 10 \mathrm{mg}$ per day prednisolone or equivalent) is associated with hospitalization for severe COVID-194.
} 
Finally, the first publication from the COVID-19 Global Rheumatology Alliance (C19-GRA) examined factors associated with hospitalization among 600 cases of COVID-19 in patients with rheumatic diseases ${ }^{4}$. C19-GRA hosts an international database aiming to capture detailed data from rheumatology providers on COVID-19 outcomes in patients with rheumatic diseases, in order to address the knowledge gap regarding factors associated with severe disease. Since launching in March 2020, >5,000 cases globally have been reported via its European and global registries ${ }^{10}$. Of the 600 cases included in the analysis by Gianfrancesco et al. ${ }^{4}, 277$ (46\%) required hospitalization. As in the general population, older age and the presence of additional underlying health conditions were factors associated with hospitalization. Use of hydroxychloroquine was not associated with hospitalization (adjusted odds ratio (OR) 0.94 ; 95\% CI $0.57-1.57$ ) but use of high-dose glucocorticoids ( $\geq 10 \mathrm{mg}$ per day of prednisolone-equivalent) was (adjusted OR 2.05; 95\% CI 1.06-3.96). The study also included a preliminary analysis of DMARD exposure and found that compared with patients who were not receiving DMARDs, patients receiving biologic DMARDs (with TNF inhibitors being the most commonly prescribed) were less likely to be hospitalized (adjusted OR 0.46; 95\% CI 0.22-0.93). Owing to the design of the C19-GRA database, it is not possible to conclude whether this observation is attributable to a higher than expected mortality rate in patients not receiving DMARDs or to a protective effect of biologic DMARDs. Patients receiving certain therapies, such as biologic DMARDs, might also be followed more closely in rheumatology clinics and, therefore, mild cases might be more likely to come to the attention of rheumatologists. Patients seen less frequently in rheumatology clinics might only come to the attention of the rheumatologist following hospitalization. Like the Williamson et al. ${ }^{2}$ and Simon et al. ${ }^{3}$ studies, interpretation of the findings of this study ${ }^{4}$ in causal terms should be avoided.

Many questions about COVID-19 in patients with rheumatic diseases remain unanswered. Further studies are required to understand the differential risk between rheumatic diseases, the individual risk associated with use of the various classes of DMARDs, as well as the long-term effects of COVID-19 in this population, in order to advise on future

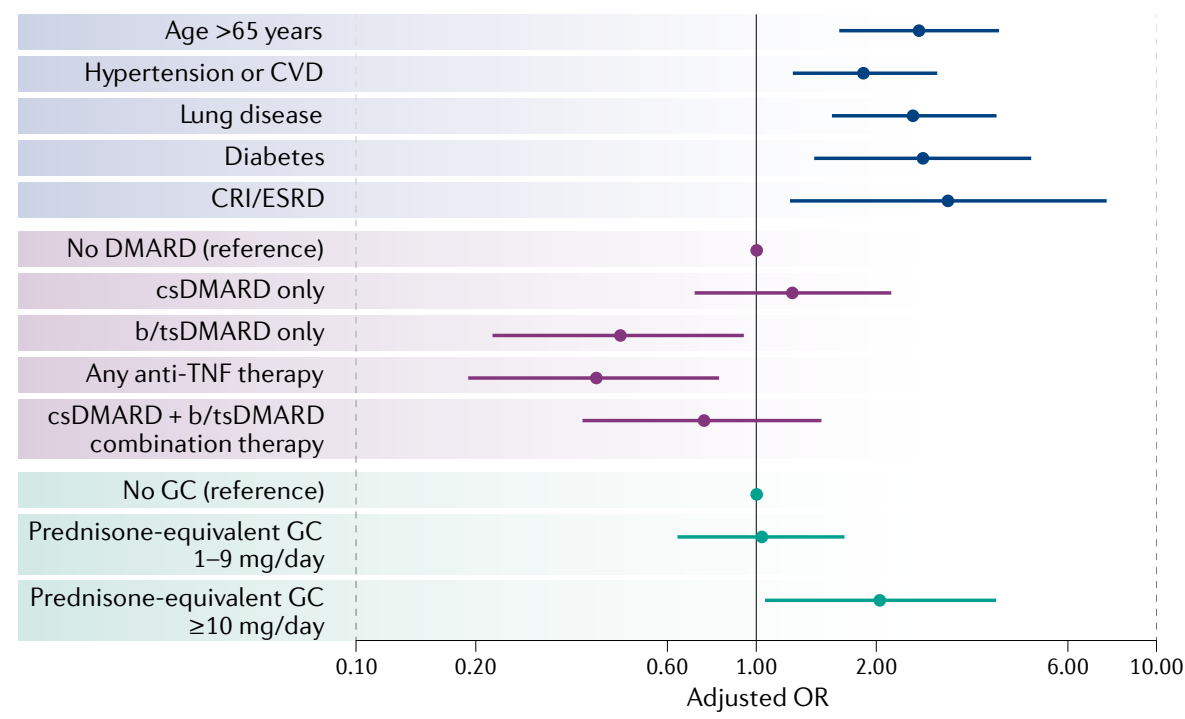

Fig. 1 | Factors associated with hospitalization for COVID-19 infection. This graph visualizes data from 600 patients with rheumatic diseases recorded in the COVID-19 Global Rheumatology Alliance international physician registry, reported by Gianfrancesco et al. ${ }^{4}$. Associations between the various factors and odds of hospitalization were estimated using multivariable-adjusted logistic regression and reported as odds ratios (ORs) with 95\% confidence intervals. b/tsDMARD, biologic/ targeted synthetic DMARD; CRI, chronic renal insufficiency; csDMARD, conventional synthetic DMARD; CVD, cardiovascular disease; ESRD, end-stage renal disease; GC, glucocorticoid. social behaviour or treatment decisions. It is early days and as more cases (unfortunately) accrue, we will continue to learn more about this novel infection.

Kimme L. Hyrich (D) ${ }^{\otimes}$ and Pedro M. Machado iD ${ }^{2}$

${ }^{1}$ Centre for Epidemiology Versus Arthritis, The University of Manchester, Manchester Academic Health Science Centre, Manchester, UK.

${ }^{2}$ Centre for Rheumatology \& Department of Neuromuscular Diseases, University College London, London, UK.

凶e-mail:kimme.hyrich@manchester.ac.uk https://doi.org/10.1038/s41584-020-00562-2

1. World Health Organization. WHO Coronavirus Disease (COVID-19) Dashboard, https://covid19.who.int/ (2020).

2. Williamson, E. J. et al. Factors associated with COVID-19-related death using OpenSAFELY. Nature 584, 430-436 (2020).

3. Simon, D. et al. Patients with immune-mediated inflammatory diseases receiving cytokine inhibitors have low prevalence of SARS-CoV-2 seroconversion. Nat. Commun. 11, 3774 (2020).

4. Gianfrancesco, M. et al. Characteristics associated with hospitalisation for COVID-19 in people with rheumatic disease: data from the COVID-19 Global Rheumatology Alliance physician-reported registry. Ann. Rheum. Dis. 79, 859-866 (2020).

5. Sepriano, A. et al. Safety of synthetic and biological DMARDs: a systematic literature review informing the 2019 update of the EULAR recommendations for the management of rheumatoid arthritis. Ann. Rheum. Dis. 79, 760-770 (2020).

6. Siemieniuk, R. A. C. et al. Drug treatments for COVID-19: living systematic review and network meta-analysis. BMJ 370, m2980 (2020).

7. Winthrop, K. L. \& Mariette, X. To immunosuppress: whom, when and how? That is the question with COVID-19. Ann. Rheum. Dis. 79, 1129-1131 (2020).

8. Leisman, D. E. et al. Cytokine elevation in severe and critical COVID-19: a rapid systematic review, meta-analysis, and comparison with other inflammatory syndromes. Lancet Respir. Med. https://doi.org/10.1016/S2213-2600(20)30404-5 (2020).

9. Zhang, B. et al. Immune phenotyping based on the neutrophil-to-lymphocyte ratio and IgC level predicts disease severity and outcome for patients with COVID-19. Front. Mol. Biosci. 7, 157 (2020).

10. COVID-19 Global Rheumatology Alliance. Healthcare Provider Entered Registries, https://rheum-covid.org/ provider-registry-gate/ (2020).

Acknowledgements

P.M.M. is supported by the National Institute for Health Research (NIHR) University College London Hospitals Biomedical Research Centre. K.L.H. is supported by the NIHR Manchester Biomedical Research Centre and Manchester University NHS Foundation Trust.

\section{Competing interests}

K.L.H. declares that she has received consulting and speaker's fees from Abbvie and grant income from BMS, Pfizer and UCB, all unrelated to this manuscript. P.M.M. has received consulting and speaker's fees from Abbvie, BMS, Celgene, Eli Lilly, Janssen, MSD, Novartis, Orphazyme, Pfizer, Roche and UCB, all unrelated to this manuscript.

\section{Disclaimer}

The views expressed are those of the authors and not necessarily those of the National Health Service, NIHR or the Department of Health. 